\title{
Palladium Catalyzed Chemo- and Enantioselective Oxidation of Allylic Esters and Carbonates
}

\author{
Barry M. Trost, Jeffery Richardson, and Kelvin Yong \\ Department of Chemistry, Stanford University, Stanford, California, 94305-5080.
}

The palladium catalyzed asymmetric allylic alkylation of nucleophiles with allyl halides, esters, carbonates and phosphonates provides an efficient and atom-economical access to a variety of useful chiral and achiral compounds. ${ }^{1}$ The use of nitronates as nucleophiles in allylic alkylation typically results in $C$-alkylation to afford synthetically useful nitroalkanes. ${ }^{2}$ However, by tuning the steric and electronic properties of the nitronate, we have discovered that $O$ alkylation can be favored and, thus, by the fragmentation mechanism shown in Scheme 1, provide a novel access to $\alpha, \beta$-unsaturated carbonyl compounds. The use of nitronates as nucleophilic oxidants for benzyl halides has been known for a long time, ${ }^{3}$ although seldom utilized in synthesis.

Nitronate 1 was chosen so as to disfavor $C$-alkylation by placing sterically encumbering groups in the adjacent positions as well as to favor $O$-alkylation electronically by minimizing disruption of conjugation in the transition state. It was generated in situ by deprotonation of the corresponding nitrocompound using KHMDS, ${ }^{4}$ which itself was generated by mixing KH and freshly distilled HMDS in the reaction vessel prior to addition of the nitrocompound.

Our studies began with the attempted oxidation of methyl 5-(benzoyloxy)-3-cyclohexene-1carboxylate, where only moderate conversion to the enone could be obtained in $8 \mathrm{~h}$ using $\mathrm{PPh}_{3}$ as ligand. Upon screening a small number of other leaving groups we discovered that the 3,5-dinitrobenzoate derivative was a significantly better substrate, affording the enone in essentially quantitative yield in less than $1 \mathrm{~h}$ (Table 1, entry 1 ). A small screen of other phosphines showed that $\mathrm{PPh}_{3}$ was the best ligand for this process and that bidentate ligands resulted in prolonged reaction times.

A variety of substrates bearing this activated leaving group were prepared and tested (Table 1) and the inherent chemoselectivity of this process towards allylic leaving groups was demonstrated. For example, a good leaving group that does not occupy an allylic position is undisturbed (Table 1, entry 7), and a variety of functional groups that might otherwise be affected by other commonly utilized oxidants also remain unchanged by this oxidation protocol. Tertiary amines (Table 1, entry 2), thioethers (Table 1, entry 3), and unprotected primary alcohols (Table 1, entry 9) are all comfortably tolerated with no occurrence of undesired oxidation. Acyclic substrates (e.g. Table 1, entries 11-12) can also be oxidized to the corresponding enones, although racemic ligand 2 appears to be more effective than $\mathrm{PPh}_{3}$ in the case studied.

The ability of chiral ligands to mediate allylic alkylation reactions and impart high levels of enantioselectivity in the products is one of the most appealing aspects of these processes. The desymmetrization of meso substrates as well as the dynamic kinetic asymmetric transformations (DYKATs) of racemic substrates with alcohols, amines, phthalimide and 
acetoacetates using ligands such as $\mathbf{2}$, have been shown to be both highly efficient and enantioselective for a broad range of substrates. ${ }^{1}$ Applying our chiral ligands to the desymmetrization of meso compounds to generate chiral enones was first studied (Table 2, entries 1-3). The five- and six-membered rings performed well affording the desired products in good yields and excellent enantioselectivity. The seven membered ring was significantly more sluggish and lower yielding, although the enantioselectivity of the process remained excellent.

Cyclopentenones differing only in the oxygen protecting group to that prepared in Table 2, entry 1 are common intermediates in the synthesis of prostaglandins and their analogues. ${ }^{5} \mathrm{~A}$ similarly close analogue of 4-oxo-2-cyclohexenyl benzoate (Table 2, entry 2) was recently employed in a partial synthesis of superstolide A. ${ }^{6}$ In both cases, these enones were prepared by enzymatic desymmetrization of a meso ester followed by a number of subsequent transformations. ${ }^{7}$ Our new oxidation method affords closely related products, which are readily available in either enantiomeric form, in just one step from almost identical starting materials.

For chiral substrates that lead to chiral products (entries 4-8) via a symmetrical $\pi$-allyl palladium intermediate in a DYKAT process, the yields and enantioselectivities were uniformly high although with some substrates the reactions became more sluggish than with $\mathrm{PPh}_{3}$ and did not reach completion.

Kinetic resolution is also possible using this protocol (Table 2, entry 10) and the conversion of the carbonate can be stopped at around $50 \%$ to afford the starting material in a highly enantioenriched state.

To demonstrate the further synthetic utility of this method, the synthesis of $\gamma$-substituted enone 4, was undertaken. This material is an intermediate in Bäckvall's synthesis of paenilactone A and was previously prepared in 3 steps from the bis-acetate. ${ }^{8}$ Palladium catalyzed malonate addition led to 3 in $68 \%$ yield and excellent enantioselectivity (Scheme 2). Oxidation using the newly developed conditions and $\mathrm{PPh}_{3}$ as ligand afforded 4 in good yield while retaining the high levels of enantioenrichment.

In conclusion, we have demonstrated a new and highly chemoselective method for the oxidation of allylic esters and carbonates. We have also shown that the reaction can be highly enantioselective for the oxidation of meso compounds and chiral substrates that proceed via symmetrical $\pi$-allyl Pd complexes. For chiral materials that do not proceed through such symmetrical Pd complexes, kinetic resolution is also possible. It should be noted that the nitroalkane $\mathbf{6}$ readily derives from the oxidation of the corresponding oxime (5) by oxidation with sodium perborate. ${ }^{4}$ Thus, recycling of the oxime by-product can enhance the efficiency of this method.

\section{Supplementary Material}

Refer to Web version on PubMed Central for supplementary material.

\section{Acknowledgement}

We thank the National Science Foundation and National Institutes of Health, GM33049, for their generous support of our programs. Mass Spectra were provided by the Mass Spectrometry Regional Center of the University of California-San Francisco, supported by the NIH Division of Research Resources.

\section{References}

(1). Trost BM. J. Org. Chem 2004;69:5813-5837. [PubMed: 15373468] 
(2). Trost BM, Surivet J-P. Angew. Chem. Int. Ed 2000:3122-3124.

(3). Hass HB, Bender ML. J. Am. Chem. Soc 1949;71:1767-9.andHass HB, Bender ML. Org. Syn 1950;30:99-101.

(4). Generation of Potassium nitronate 1.

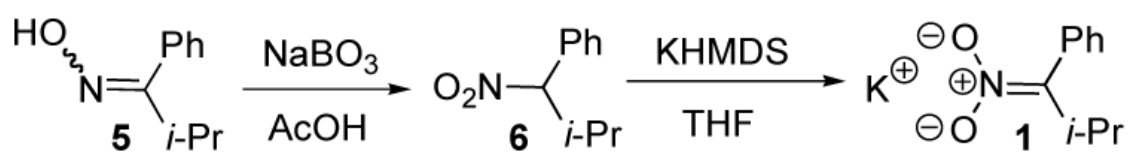

It was necessary to generate KHMDS in situ to avoid reproducibility issues encountered using stock and commercially available solutions.

(5). Noyori R, Suzuki M. Angew. Chem. Int. Ed 1984;23:847-876. b. Jung ME, Kers A, Subbanagounder G, Berliner JA. Chem. Comm 2003;2:196-197. [PubMed: 12585388]

(6). Hua Z, Yu W, Su M, Jin Z. Org. Lett 2005;7:1939-1942. [PubMed: 15876024]

(7). Paquette LA, Heidelbaugh TA. Org. Syn 1996;73:44-48.

(8). Jonasson C, Roenn M, Bäckvall J-E. J. Org. Chem 2000;65:2122-2126. [PubMed: 10774035] 


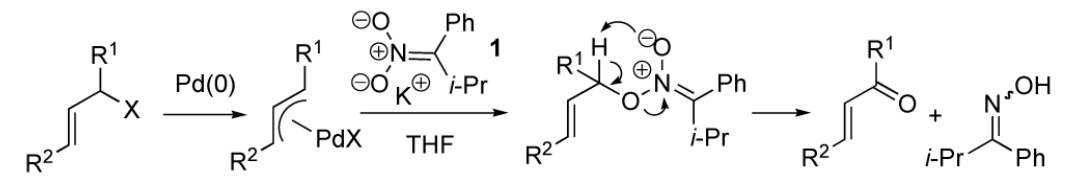

Scheme 1.

Mechanism of Allylic Oxidation 


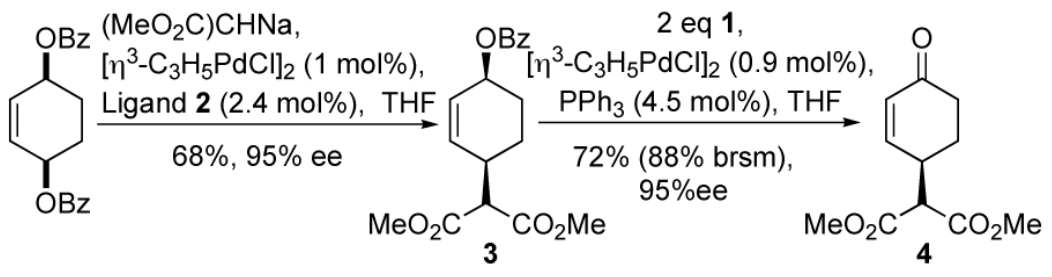

Scheme 2.

Synthesis of Bäckvall's paenilactone A intermediate 
Table 1

Oxidation of Allylic Esters and Carbonates

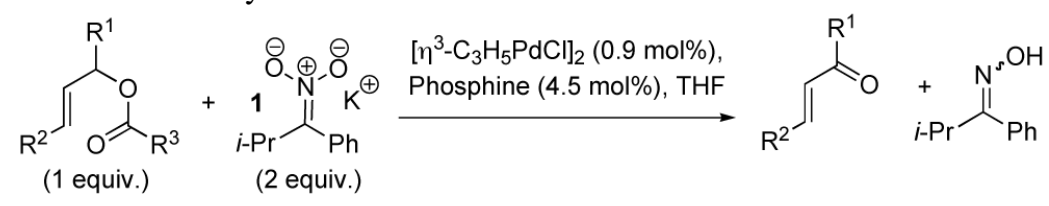

\begin{tabular}{|c|c|c|c|c|}
\hline Entry & Substrate & Product & Phosphine & Yield (time) \\
\hline $\begin{array}{l}1 \\
2 \\
3\end{array}$ & & & $\begin{array}{c}\mathrm{PPh}_{3} \\
\mathrm{PMePh}_{2} \\
\text { dppf }\end{array}$ & $\begin{array}{c}100 \%(<1 \mathrm{~h}) \\
95 \%(<1 \mathrm{~h}) \\
80 \%(2 \mathrm{~h})\end{array}$ \\
\hline 4 & & & dppb & $73 \%(26 \mathrm{~h})^{a}$ \\
\hline 5 & & & $\mathrm{PPh}_{3}$ & $92 \%(1 \mathrm{~h})$ \\
\hline 6 & & & $\mathrm{PPh}_{3}$ & $96 \%(>1 \mathrm{~h})$ \\
\hline 7 & & & $\mathrm{PPh}_{3}$ & $88 \%(>1 \mathrm{~h})$ \\
\hline 8 & & & $\mathrm{PPh}_{3}$ & $\mathrm{R}=\mathrm{OTBS}, 95 \%(2 \mathrm{~h})$ \\
\hline 9 & & & $\mathrm{PPh}_{3}$ & $\mathrm{R}=\mathrm{OH}, 82 \%(2 \mathrm{~h})$ \\
\hline 10 & & & $\mathrm{PPh}_{3}$ & $71 \%(3 \mathrm{~h})^{b}$ \\
\hline 11 & & & $\mathrm{PPh}_{3}$ & $53 \%(2.5 \mathrm{~h})$ \\
\hline 12 & & & $r a c-2$ & $97 \%(4 \mathrm{~h})$ \\
\hline
\end{tabular}

$a_{95 \% \mathrm{brsm} .}$

${ }^{b}$ Volatile material. Actual yield may be higher. 


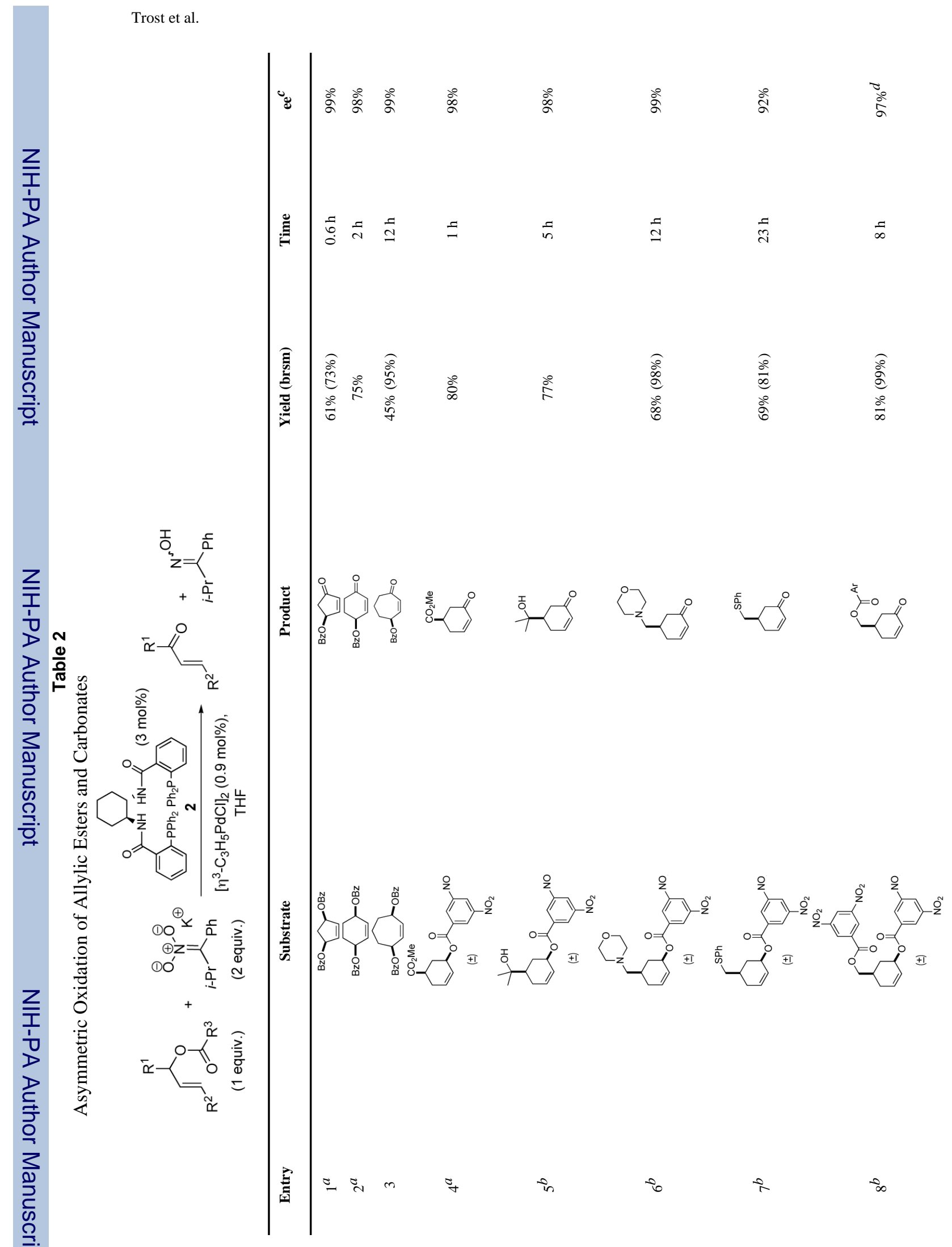

J Am Chem Soc. Author manuscript; available in PMC 2008 September 6. 
Trost et al.

Page 8

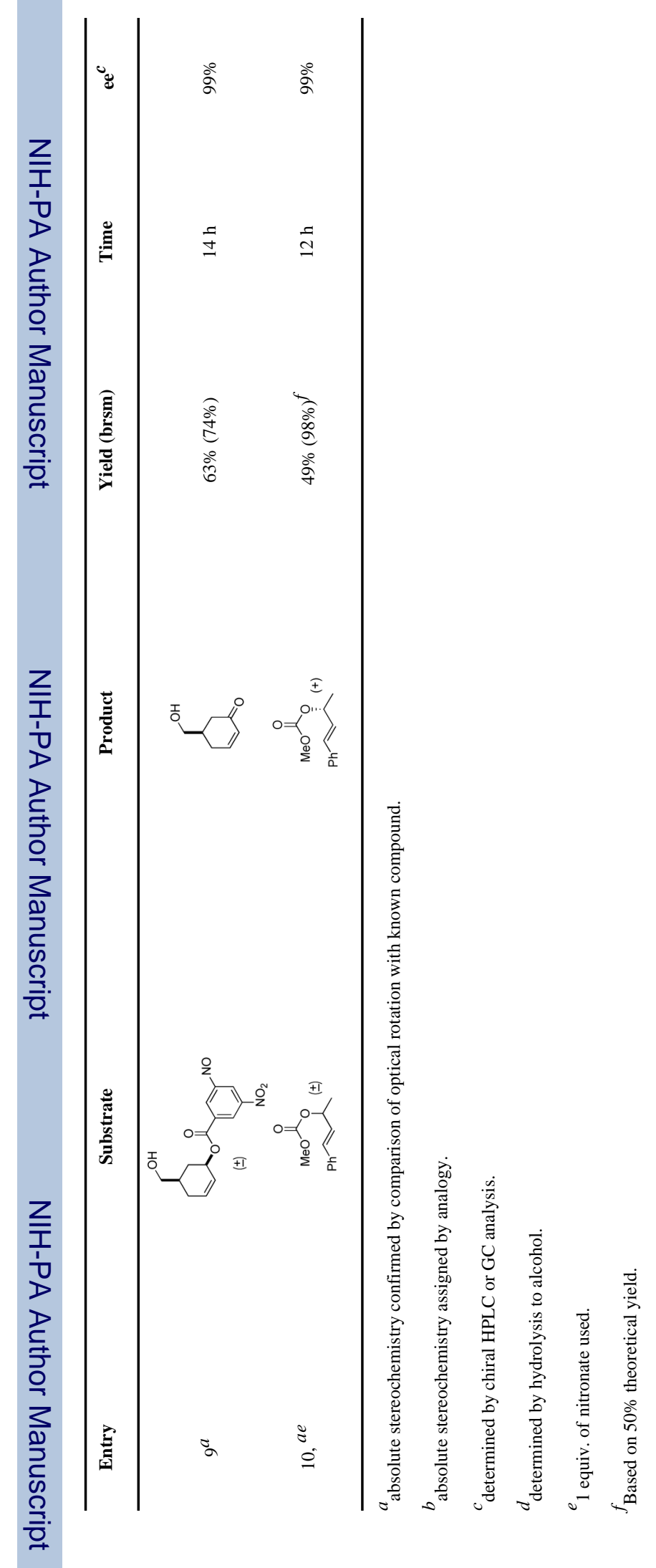

J Am Chem Soc. Author manuscript; available in PMC 2008 September 6. 